\title{
Adultez Emergente y características culturales de la etapa en universitarios chilenos
}

\author{
Emerging Adulthood and Cultural Characteristics of the Stage in Chilean University \\ Students
}

\author{
Ana Barrera-Herrera \\ Eugenia V. Vinet \\ Universidad de La Frontera, Chile
}

Rec (30 de junio de 2016) Acept (22 de febrero 2017)

\begin{abstract}
Resumen
La Adultez Emergente es un periodo de vida entre los 18 y 29 años, culturalmente construido y no de carácter universal. Ante la escasez de estudios en esta etapa en Chile, el objetivo de esta investigación fue caracterizar la Adultez Emergente, y determinar sus rasgos más relevantes en universitarios chilenos, a través de una aproximación cualitativa, desde los protagonistas. Seis grupos focales conformados por 60 universitarios a nivel nacional analizaron los ítems del Inventario de Dimensiones de Adultez Emergente o discutieron sus creencias sobre tres áreas definidas a priori: Identidad y Autoconcepto, Relación con los padres y salida del hogar, y Amor y sexualidad. Se evidencia la presencia de la Adultez Emergente en universitarios, pues las características centrales de la teoría están presentes en este grupo, y se obtiene evidencia de aspectos de carácter cultural propios de los universitarios chilenos, relacionados a la importancia de los vínculos familiares y sociales.

Palabras clave: Adultez emergente, Psicología del Desarrollo, Universitarios, Cultura.
\end{abstract}

\begin{abstract}
Emerging Adulthood is a period of life between 18 and 29 years old, culturally constructed and not universal. Given the paucity of studies about this developmental stage in Chile, the objective of this research was to characterize Emerging Adulthood and determine its most important features in Chilean university students using a qualitative approach, focused at the protagonists themselves. Six focus groups formed by 60 university students revised the items of the Inventory of Dimensions of Emerging Adulthood (IDEA) or discussed their beliefs about three areas defined a priori: Identity and self-concept, Relationship with parents and leaving home, and Love and sexuality. The presence of the Emerging Adulthood stage in university students is evident since its central features are present in this group; also there is evidence of others aspects that belong to cultural characteristics of the own Chilean university students which are related to the importance given to the family and social relationships.

Key words: Emerging Adulthood, Developmental Psychology, University students, Culture.
\end{abstract}

\footnotetext{
Correspondencia: La correspondencia relativa a este artículo debe ser enviada a Ana Barrera-Herrera. Departamento de Psicología, Universidad de La Frontera, Avenida Francisco Salazar 01145. Temuco, Chile. E-mail: ana.barrera@ufrontera.cl.

Este estudio recibió financiamiento de la Dirección de Investigación de la Universidad de La Frontera (Pro. DIUFRO DI14-2005) y del Proyecto FONDECYT N ${ }^{\circ} 1150095$.
} 


\section{Introducción}

En Chile, según las proyecciones de los últimos datos recabados por la Séptima Encuesta Nacional de la Juventud (INJUV, 2012), el número de jóvenes entre el rango de 15 a 29 años para el año 2015, supera los cuatro millones, un número no menor de personas que es sujeto de estudio en una gran diversidad de variables relacionadas con salud, vivienda, participación social y educación. Específicamente, en el ámbito educativo, un grupo que llama la atención debido a la contingencia del Movimiento Estudiantil es el de los estudiantes universitarios chilenos. Ellos, según datos del Consejo Nacional de Educación (CNED, 2015) alcanzan a 1.152.125 estudiantes matriculados en pregrado solo en el año 2015 en instituciones de Educación Superior, incluyendo Centros de Formación Técnica, Institutos Profesionales y Universidades.

Según el CNED (2015) solo a nivel de Universidades, Chile cuenta con más de 640.000 estudiantes matriculados, los cuales están en un periodo de formación académica, preparándose para un futuro profesional cercano, que les permita conseguir un puesto laboral estable y alcanzar una mejor situación económica, variables que ellos mismos observan relacionadas con su nivel de felicidad y éxito personal (INJUV, 2016). Esta etapa de vida universitaria es un periodo de interés por sí misma, pues comprende un periodo de cinco o seis años en los cuales ocurren cambios tanto a nivel del desarrollo académico de los jóvenes como a nivel de desarrollo personal. Desde el punto de vista de la Psicología del Desarrollo, la fase universitaria coincide con el periodo de vida llamado Adultez Emergente, etapa situada entre los 18 y los 29 años donde lo más característico es que los jóvenes que transitan por ella no se ven como adolescentes, y la mayoría de ellos tampoco siente que ha alcanzado la adultez (Arnett, 2000, 2004, 2012).

La Adultez Emergente (AE) se manifiesta como un fenómeno cultural en sociedades industrializadas a partir de los cambios sociodemográficos de las últimas décadas, tales como el aumento del acceso a la educación universitaria, el aumento de la fuerza laboral, y la postergación de hitos como el matrimonio y tener hijos (Arnett, 2000). A este respecto, Chile no ha estado ajeno a estos cambios sociodemográficos, pues como lo reportan varios estudios (Cerda, 2008; Dávila y Ghiardo, 2012; Instituto Nacional de Estadísticas, 2011), en las últimas décadas se ha producido un aumento de los años de escolaridad, un mayor acceso a la educación superior, y un mayor acceso al trabajo por parte de las mujeres, disminución de la tasa de hijos, y un aumento sostenido de la edad para contraer matrimonio, factores que indican una tardía entrada a la adultez, confirmando la existencia de la $\mathrm{AE}$ como un periodo de vida evolutivamente relevante a nivel país.

Este periodo único y heterogéneo, presenta características específicas que pueden variar dependiendo de la cultura en la cual se desarrolle el individuo (Arnett, Kloep, Hendry, y Tanner, 2011). En Estados Unidos se han descrito cinco características distintivas de esta etapa: (a) Exploración de la identidad; (b) Inestabilidad; (c) Gran optimismo y posibilidades; (d) Estar centrado en sí mismo; y (e) Ser una etapa de sentirse "en el medio" entre la adolescencia y la adultez (Arnett, 2000, 2004). Estas cinco características describen a los jóvenes norteamericanos; sin embargo, dado que la teoría afirma que esta etapa de vida es un período culturalmente construido (Arnett, 2000, 2004), se plantea la posibilidad de que la forma en que esta se desarrolle en cada sociedad podría tener características culturales distintivas, dependiendo de cuál grupo de jóvenes sea objeto de estudio.

Respecto de los jóvenes universitarios chilenos, existen variados estudios, los cuales en su mayoría dan cuenta de prevalencias de psicopatología, o estadísticas generales referidas a datos demográficos de este grupo (Baader et al., 2014; Casas et al., 2014; INJUV, 2012; Mediano-Stoltze, Repetto, y Molina, 2013; Micin y Bagladi, 2011; Morales et al., 2011, entre otros). Sin embargo, hasta ahora, no se ha analizado si los universitarios chilenos poseen las características centrales de la $\mathrm{AE}$, ni se ha descrito cómo transitan por esta etapa de vida, o si existen diferencias en las características representativas de la AE chilena respecto a esta etapa en otras culturas, como por ejemplo, la anglosajona, en términos de procesos psicológicos afectivos y relacionales por los que atraviesan los jóvenes en el ámbito universitario. Ante esta necesidad, el objetivo general de este estudio fue caracterizar la etapa de Adultez Emergente en jóvenes chilenos universitarios, de 18 a 29 años, desde los propios protagonistas. Para este propósito se definieron dos objetivos específicos: (a) Identificar las creencias culturales de los jóvenes respecto a la experiencia de AE en la etapa universitaria; y (b) Describir los desafíos y oportunidades de esta etapa en universitarios chilenos considerando las áreas de Identidad y Autoconcepto, Relación con los padres y salida del hogar, y Amor y sexualidad, relevantes en la cultura local (INJUV, 2016; Rivera, Cruz, y Muñoz, 2011; Véliz-Burgos y Apodaca, 2012). 


\section{Método}

\section{Participantes}

A través de un muestreo no probabilístico, tipo bola de nieve, se obtuvo una muestra de 60 jóvenes universitarios pertenecientes a universidades estatales de Santiago, Talca y Temuco (20 estudiantes por cada ciudad), los cuales participaron en grupos focales conducidos por miembros del equipo de investigación.

El $68,3 \%$ de la muestra fueron mujeres y la media de edad del grupo total fue de 22 años, con una DT de 2,21 años. Un $83,3 \%$ de los universitarios vivían con sus padres, y la mayor parte de ellos $(93,3 \%)$ lo hacía en zonas urbanas. El $55 \%$ de los participantes declara pertenecer al Nivel Socio Económico (NSE) medio, y un $37,7 \%$ al NSE medio-bajo. En relación con la etnia, solo un $6,7 \%$ se declara Mapuche.

En cuanto a otras variables de interés para el periodo de $\mathrm{AE}$, solo un $30 \%$ de la muestra compatibiliza sus estudios con actividades laborales, y un $48,3 \%$ se encuentra en una relación de pareja. Finalmente, frente a la pregunta “'Sientes que has alcanzado la plena adultez?”, un 59,3\% declara que en algunos aspectos sí, mientras que en otros no, un $22 \%$ sí siente que ha alcanzado la adultez, mientras que un $18,6 \%$ responde negativamente a esta pregunta. El porcentaje mayoritario refleja la sensación de los jóvenes de "sentirse en el medio", característica representativa de este periodo de vida.

\section{Procedimiento}

Los participantes fueron contactados a través de informantes claves de las universidades de interés (profesores o directores de carrera), y por difusión masiva a través de afiches. Previo a su participación, firmaron un consentimiento informado donde se explicitaba que la actividad no tendría consecuencias negativas para su integridad física y psicológica, y que su participación era anónima y voluntaria. Este documento fue visado y aprobado por el Comité Ético Científico de la Universidad de La Frontera, al igual que todos los procedimientos descritos en esta investigación.

En cada ciudad se realizaron dos tipos diferentes de grupos focales. El Grupo Focal 1 (IDEA), tuvo por objetivo explorar qué tan representativos/relevantes eran los ítems de la versión original del Inventario de Dimensiones de Adultez Emergente (IDEA; Reifman, Arnett, y Colwell, 2007) respecto a su etapa de vida actual, desde la perspectiva de los mismos universitarios. Para ello se les presentó como insumo para orientar la discusión la versión traducida del IDEA (Pérez, Cumsille, y Martínez, 2008), junto a una sección de preguntas abiertas para que ellos propusieran nuevas áreas de interés no incluidas en el instrumento original.

El Grupo Focal 2 (Creencias culturales) exploró, a través de preguntas abiertas, las creencias culturales de los participantes respecto a su experiencia en la etapa de vida actual sin explicitar directamente el constructo evaluado, por ser un término poco conocido. En esta discusión se consideraron las temáticas de Identidad y Autoconcepto, Relación con los padres y salida del hogar, y Amor y sexualidad, áreas escogidas a priori y no cubiertas por el IDEA original que parecen ser evolutivamente relevantes en una sociedad como la chilena que conserva aspectos colectivistas sobretodo en el ámbito de las relaciones familiares y sociales (Novoa y Barra, 2015; Rojas-Méndez, Coutiño-Hill, Bhagat, y Moustafa, 2008).

\section{Instrumentos}

Cuestionario sociodemográfico. Fue aplicado en todos los grupos focales para indagar variables que permitieran caracterizar la muestra, en términos de edad, género, nivel socioeconómico, región de residencia, entre otros.

Inventario de Dimensiones de Adultez Emergente, IDEA (Reifman et al., 2007). Como se señaló, en el Grupo Focal 1 se utilizó como insumo el instrumento compuesto por 31 ítems, los cuales consisten en preguntas, con un formato de respuesta Likert, que va desde "Fuertemente en desacuerdo" a "Fuertemente de acuerdo". En este estudio se utilizó la versión chilena del IDEA (Pérez et al., 2008). En el Grupo Focal 2 no hubo un instrumento estructurado, solo se estimuló la participación con preguntas abiertas sobre la etapa universitaria y su paso por este periodo de vida.

\section{Plan de análisis}

La información obtenida por los dos tipos de grupos focales fue analizada utilizando el Análisis de contenido, empleando específicamente el Método de Comparaciones Constantes en el marco de la Teoría Fundamentada de Glaser y Strauss (1967). Para ello, en primer lugar, miembros del equipo de investigación realizaron la transcripción de los grupos focales. Luego, de forma individual procedieron a realizar la codificación de cada trascripción, utilizando el Software ATLAS.ti. Posteriormente, se triangularon las codificaciones, obteniendo como resultado un total de 301 códigos, que fueron organizados en cinco grandes dimensiones, esto es: "Características de la AE para los universitarios 
chilenos", "Relación con los padres en la AE", "Salida del hogar familiar en la AE", "Relación de pareja", y por último "Proyecciones en la relación de pareja". La organización de estas cinco dimensiones y el análisis de reflexiones teóricas y empíricas resultantes, permitieron responder los objetivos de investigación, los cuales serán expuestos a continuación.

\section{Resultados}

En esta sección se explicitarán en primer lugar tres características generales de la $\mathrm{AE}$ coincidentes con la conceptualización general de la teoría, y posteriormente se expondrán los hallazgos relacionados con tres aspectos más específicos de esta etapa, propios de la cultura chilena.

\section{Adultez Emergente en la etapa universitaria}

Uno de los hallazgos más importantes corresponde a que la mayor parte de las características generales de la AE (etapa de exploración de la identidad, etapa de inestabilidad frente a los cambios y decisiones futuras, etapa de estar centrado en sí mismo, y de sentirse "en el medio") fueron reconocidas como presentes en este periodo por la totalidad de los participantes, lo cual confirma la existencia del fenómeno de AE en este grupo, desde el análisis de las respuestas entregadas por los propios protagonistas.

Para los fines de este estudio, solo se reportarán las tres características de esta etapa que tuvieron mayor relevancia al momento de describir la fase universitaria. Estas son: $\mathrm{La}$ AE como a) un periodo de exploración, b) un periodo para construir la identidad, y c) un periodo para sentirse en el medio, y serán tratadas a continuación.

En primer lugar, los universitarios ven esta etapa como un periodo de exploración, el cual incluye una fase crucial para la toma de decisiones sobre, por ejemplo: la carrera escogida, el vivir solos, o participar de actividades en la universidad. En cada contenido sujeto a decisiones el estudiante debe evaluar distintas opciones y decidir prioridades de forma gradual y con responsabilidad, teniendo como marco de referencia sus creencias y valores. Tales decisiones, según su punto de vista, guían su proyección laboral, o personal.

También señalan que a la par de explorar sus decisiones, el cursar una carrera universitaria les permite desarrollar competencias genéricas, como las habilidades interpersonales de empatía y tolerancia, habilidades de comunicación, capacidad de corregir errores y pensamiento crítico. Este desarrollo de competencias propicia la exploración de sus ideologías y creencias, reforzando, algunas ocasiones, las creencias que provienen de su núcleo familiar, mientras que en otras desafían los valores y creencias familiares, pues al enfrentar la diversidad cultural y social universitaria, adquieren nuevas creencias sobre su carrera, las relaciones de pareja, y comienzan a formalizar sus ideologías en relación a tópicos como, por ejemplo, la política y la religión.

\section{Mujer, 20 años: "Se han reforzado algunas (creencias) $y$ otras se han debilitado, porque de todas maneras hay un entorno donde hay múltiple diversidad, entonces hay múltiples opiniones, múltiples ideas, múltiples creencias y compartiendo con diferente gente uno puede adoptar algunos puntos de vista o rechazar otros, ir reforzando los propios... y otros que he ido desechando".}

Esta exploración en el periodo universitario, también trae consigo posibilidades y oportunidades en áreas variadas. Destacan la libertad general, expresada como mayor libertad para equivocarse al momento de explorar y evaluar opciones, pues al no tener las responsabilidades de un adulto (trabajo, hogar e hijos), se reducen las consecuencias o el impacto de sus decisiones. También esta libertad permite que los jóvenes exploren en el consumo de sustancias, como alcohol y/o marihuana en fiestas universitarias, explicitando que en la universidad tendrían un mayor acceso a drogas legales e ilegales.

\begin{abstract}
Mujer, 21 años: "Entonces tú al entrar a la universidad tienes la posibilidad de explorar en otras cosas también, o sea, por ejemplo, yo conocí el teatro, nunca en mi vida había sabido que habría podido servir un poquito en eso y entré... pero igual uno explora en otras cosas ¿me entiendes?, como en sustancias ilegales".
\end{abstract}

En segundo lugar, los adultos emergentes universitarios señalan que este es un periodo para construir la identidad, pues se propicia el descubrimiento del sí mismo, transitando hacia una conformación progresiva de la identidad, a medida que van madurando y alcanzando mayor independencia y autonomía en las áreas emocional, económica y académica. Específicamente, el logro progresivo de mayor estabilidad, o autorregulación tanto emocional como académica, además de la adquisición de mayores responsabilidades (tales como manejo del dinero, responsabilidad académica, responsabilidad laboral si trabajan, responsabilidad por sí mismos, y ciertamente una responsabilidad social), les otorga un 
sustento que les permite definirse y conocerse en cuanto a sus capacidades y potencialidades.

Hombre, 22 años: "Yo creo que uno descubre las potencialidades que tiene, una búsqueda de habilidades... uno sale de la media y cree que lo tiene todo súper claro y comienza a avanzar y se da cuenta que se desestructura todo y uno no vuelve atrás a ser, entonces es como una búsqueda de identidad. Es como saber que tu quizás eres bueno para esto y quizás estas yendo por esta trayectoria y te equivocaste y puedes volver y tomar otra opción. En cierta forma nos vamos validando a través de la experiencia, por lo menos en mi propia vida yo lo vivencié así".

En este proceso de construcción de identidad y autodescubrimiento, existirían facilitadores y obstaculizadores. Factores como la diversidad del contexto, las situaciones de toma de decisiones y de manejo de la presión frente a dificultades, el estar en pareja y el vivir solos, propiciarían el autoconocimiento y acelerarían la conformación de la identidad. Sin embargo, estos procesos pueden verse limitados por factores como dependencia de los padres, falta de interés por explorar, inseguridad o dependencia de las opiniones de los demás por sobre las propias.

La tercera característica destacada por los universitarios es que esta etapa es un periodo para sentirse "en el medio". Un 70\% de los participantes se percibe como adolescentes o en un periodo de transición. Percibirse como adolescentes implica la percepción de la propia impulsividad, su inmadurez o la desorientación respecto a su elección vocacional, y/o a su presente o su futuro. Quienes se perciben en un periodo de transición, observan que están en camino a convertirse en adultos, bajo un periodo de cambios en su identidad, ideologías y relaciones; además consideran que se encuentran en un periodo de reflexión, esbozando los primeros trazos en la planificación de su proyecto de vida. Quienes se perciben como adultos bordean un escaso $20 \%$, y generalmente son quienes poseen un trabajo, independencia económica, o tienen responsabilidades parentales y/o domésticas.

Mujer, 23 años: "Me siento adulta en algunos aspectos y en otros no. Por eso siento que en la independencia en términos de espacio no soy una adulto, sino una cabra chica todavía de cinco años para mis papás, por eso como que uno ya se proyecta $y$ dice "el otro año me voy a poner las pilas, me voy a pagar el arriendo, el agua, aunque gane 200 lucas como trabajadora social", pero (a la vez) no quiero sentirme mujer y grande, y que me la puedo sola...".

$\mathrm{Al}$ explorar las razones que favorecerían que los jóvenes se "sientan en el medio", los participantes plantean que la dependencia emocional y financiera de sus padres es una de las razones. Agregan que no hay un autoconocimiento totalmente logrado, sino en proceso de construcción. Además, y quizás una de las razones de mayor peso para "sentirse en el medio", es que la mayor parte de los jóvenes posee una visión un tanto negativa de la adultez; para ellos un adulto es alguien responsable, independiente financiera y emocionalmente de los padres, autónomo, capaz de proyectarse y establecer metas, alguien que tiene un trabajo estable y ha formado una familia; sin embargo, esta percepción está asociada a grandes responsabilidades y problemas. Por tanto, para ellos, este es un "periodo para saber lo que es ser adulto, pero no para serlo". Así, pareciera que la postergación del paso a la adultez guarda relación con una visión negativa y desafiante del futuro.

Además de las características generales abordadas en los párrafos precedentes, a continuación se reportan tres temas que corresponden a características aparentemente culturales de la $\mathrm{AE}$ en universitarios chilenos.

\section{Relación con los padres y la salida del hogar}

Los participantes señalaron una amplia variedad de características para referirse a la relación afectiva con sus padres: algunos reportaban una buena relación con sus padres, otros una relación regular, sin mayores inconvenientes, y en menor medida otros describieron su relación actual como distante o en crisis. No obstante esta variada gama de relaciones afectivas, un aspecto ampliamente compartido por la totalidad de los participantes, es que los jóvenes observaban cambios en la relación con sus padres, a partir del ingreso a la Universidad. Entre los cambios positivos destacan la mayor independencia y libertad que han obtenido, en cuanto a permisos, salidas, y manejo del tiempo. En el ámbito afectivo, existe una mayor cercanía emocional, y una valoración positiva, ya que reportan que sus padres los perciben más maduros y capaces.

En el lado menos amable, un porcentaje menor de jóvenes, cercano al $20 \%$, reportan que sus padres se han vuelto más estrictos y perciben un mayor control parental en los estudios, las salidas, y la asistencia a clases. Otros señalan que cuando ellos han elegido una carrera distinta a la esperada por sus padres, se produce un conflicto familiar de importancia, que muchas veces genera un distanciamiento 
afectivo. Otro aspecto negativo es la presión académica implícita o explícita por el rendimiento en la universidad, aspecto que es vivido negativamente a nivel emocional y que, en ocasiones, facilita inseguridades vocacionales o bajo desempeño académico.

Hombre, 21 años: "La presión que quizás ellos no ejercen... es cierto que ellos te apoyan, que ellos te contienen, pero uno se siente como con la presión de no decepcionarlos o hacer mal algo o lo que sea".

Dado que la mayoría de los participantes vivían en el hogar familiar $(83,3 \%)$, la convivencia familiar fue un tema destacado que contiene aspectos positivos y negativos. Entre los positivos, se destaca la tranquilidad y comodidad de vivir en el hogar familiar; reportan sentirse felices pues cuentan con un espacio personal, tienen compañía familiar, perciben que existe una preocupación recíproca entre ellos, y cuentan con el tiempo suficiente para preocuparse de sus deberes académicos. Entre los aspectos negativos reportan la ausencia de espacio personal o privacidad, el control o supervisión parental sobre sus conductas, decisiones o desempeño académico, el tener que asumir un rol parental con hermanos menores, y la percepción de ser una carga por continuar viviendo con los padres o ser motivo de gasto económico. Estos aspectos favorecen el deseo de independizarse en un futuro cercano, cuando egresen de su carrera profesional.

Al respecto, la mayoría de los universitarios planifica la salida del hogar familiar para el momento en que egresen de la carrera, a los 24 años aproximadamente; sin embargo, otros deciden postergar este hito hasta el momento en que ya estén establecidos laboralmente y tengan ahorros, mientras que hay quienes que aplazan este hito por ser dependientes económica y emocionalmente de los padres, o porque aún no se sienten preparados. Por tanto, el marcharse del hogar paterno se planifica en la población chilena antes de los 30 años, y son escasos los jóvenes que no desean abandonar el hogar familiar en un plazo de tiempo cercano.

Mujer, 22 años: "Una dificultad más pa' mi que pa' mis papás, es que yo ya me siento carga, yo ya quiero salir de la casa, ¿cachay?, entonces yo no sé qué pensarán ellos, ellos obviamente nunca te van a decir nada, pero yo ya siento que estoy como en la edad de independizarme".
Hombre, 20 años: "Como a los 24, porque así sería como egresar, trabajar, y tener sus lucas para poder mantenerse solo".

Por otro lado, existe un porcentaje menor de jóvenes que no viven con sus padres (16,7\%), estos han adelantado su independencia debido a cambios de ciudad por motivos académicos. Esta independencia trae consigo variados desafíos tanto emocionales como prácticos, y el contar con una red de apoyo social cercana es de vital importancia para la adaptación a ellos sobre todo en los primeros años de universidad.

\section{Amor y sexualidad}

En el ámbito de las relaciones de pareja, los participantes en general reportan que establecer una relación de pareja formal en la etapa universitaria no constituye una prioridad para la mayoría de ellos, al menos en los primeros años de universidad; prefieren explorar en relaciones informales o no tienen interés de estar en pareja. Pocos participantes explicitaron tener relaciones de pareja, aunque los datos sociodemográficos muestran que más del $40 \%$ de ellos está en pareja, con distintos grados de compromiso. En su relato, la mayoría de los jóvenes explicitaba que tenían otras prioridades, como crecer individualmente, dedicarse a la universidad, o simplemente querían estar solos.

Hombre, 21 años: "Yo creo que dentro de la universidad, uno tiene sus prioridades, y cuando uno ya sale, cambian, y entonces ahí uno evalúa si realmente la persona que está al lado tuyo cumple ciertas cosas, como para acompañarte en ese camino que viene más adelante".

Mujer, 20 años: "Hay personas que no pueden estar solos y necesitan como de esa contención y todo el apoyo, y estar ahí pololeando y todo el día, $y$ "tomemos los ramos juntos", pero ahí estamos lo otros... yo no creo que soportaría una relación en este momento de mi vida, entonces yo no me veo con alguien el resto de mi vida, yo quiero un departamento con mis cosas sola, para mi sola y no quiero estar con alguien".

Con respecto a la sexualidad fue notoria la dificultad para obtener reportes sobre esta área, pues los jóvenes mostraron reticencia y pudor para hablar del tema. 
$\mathrm{Al}$ indagar los temas de convivencia en pareja, matrimonio, $\mathrm{y}$ tener hijos los reportes fueron los siguientes. Respecto de la convivencia, un $50 \%$ de los jóvenes considera que en esta etapa universitaria no se perciben preparados para enfrentar tal desafío. El otro grupo, sí contempla la convivencia como una posibilidad de vida, pero idealmente luego de un periodo de independencia. Agregan que la convivencia es un buen ensayo para ver cómo se vive con una pareja y un paso previo al matrimonio.

Hombre, 20 años: "A mí me gustaría vivir solo en mi departamento un tiempo, y después vivir juntos, pero eso es lo que siempre he querido, salir de mi casa, mantenerme con mi propia plata, tener mis cosas solo y no sé, quizás ella igual esté viviendo sola, pero después más adelante sí, vivir juntos".

En cuanto al matrimonio, la discusión arrojó información variada. Un $60 \%$ de los participantes aproximadamente explicitaba que no tenía interés en casarse, que el matrimonio no estaba dentro de su proyecto de vida, o que preferían una celebración simbólica de compromiso, antes que una celebración legal o religiosa.

Mujer, 20 años: "Mis padres igual son separados, pero, y también el tema del matrimonio para mí, nunca fue mucho tema, porque yo siempre, independiente de que la relación de mis papás duró harto tiempo, bastante estable... pero considero que el tema como de la afectividad, el amor, no va por un contrato, entonces al final, el matrimonio como institución, a mi no me va, ni me viene".

Otros jóvenes, en menor medida (cerca del 20\%), y con algo de cautela, refirieron que se proyectaban casándose con su pareja actual o con una eventual pareja futura, puesto que creían en el amor, y obtendrían un apoyo incondicional del otro, además de seguridad económica y/o legal ante eventualidades, como muerte, cuidado de la pareja, etc.

Mujer, 21 años: "Yo siempre he considerado que voy a casarme, pero igual lo veo como algo más simbólico, porque creo que ahora, con todo lo que ha pasado por el tema de las religiones y esta casi como fobia que tiene mucha gente a ese tema, muchos han dejado de lado lo de casarse, pero solamente por el tema de las religiones en sí. Y es verdad, a mí tampoco me llamaría demasiado la atención casarme así como por un tema religioso, pero sí como algo simbólico, para demostrar que yo si elijo a esa persona, o sea, yo no estoy así como probando a ver si funciona, y si no funciona cada uno pa'su casa, no, o sea yo realmente estoy segura de que quiero estar con él y casarme".

Por último, en relación con tener hijos, los participantes en su mayoría mostraron cierta evitación y negatividad al proyectarse teniendo hijos. Explicitan marcadamente que para asumir tal desafío es necesario una serie de requisitos previos, tales como autoconocimiento, madurez, y la presencia de un disfrute individual previo, a través de la carrera, salud y viajes; además se reporta la necesidad de priorizar la estabilidad económica antes de tener hijos y el apoyo de la pareja en términos de crianza.

Hombre, 20 años: "Tener un hijo es como una responsabilidad que, la juventud como que se acorta, porque uno igual se limita a hacer cosas... pero igual, después de los treinta yo creo que igual es una edad buena, pero a mí igual me gustaría ser papá más joven, pero no tan, tan joven, no sé, como a los veintiocho".

Entre los aspectos negativos que se asociaron con el tener hijos, se encuentra la presión social y familiar por tener hijos, y el percibir esto como un desafío lleno de responsabilidades. Agregan que el tener hijos en este periodo acortaría su juventud, y les dificultaría disfrutar de esta etapa de vida. El debate sobre este tema mostró que la mayor parte de los participantes ha decidido postergar la llegada de hijos, no se proyecta en su plan de vida formando una familia o se encuentra en una etapa inicial de cuestionamientos al respecto. Finalmente, hay quienes nombraron la opción de adoptar, como una alternativa válida para ser padres.

Mujer, 23 años: "Yo creo que también está el tema de plantearse el si quiero o no ser mamá, porque a uno igual la crían como con la mentalidad, de que es parte de la vida ser mamá, pero eso, yo nunca me lo cuestioné hasta hace unos tres años atrás, y dije "¿realmente quiero tener hijos?", porque de repente uno no se cuestiona, uno piensa que es un deber como mujer y no necesariamente lo es, entonces, yo en este minuto de mi vida... me encantan los niños, tengo mucho feeling con los niños, pero no sé si quiera ser mamá". 


\section{Discusión}

La Adultez Emergente es un periodo evolutivo, culturalmente construido, que abarca las edades de 18 a 29 años, periodo que coincide con el paso por la Universidad en los jóvenes chilenos. En Chile, este periodo presenta características similares a las descritas en jóvenes estadounidenses (Arnett, 2000, 2004), siendo reconocidas las cinco características generales de la etapa, con énfasis en la $\mathrm{AE}$ como a) un periodo de exploración, $\mathrm{b}$ ) un periodo para construir la identidad, y c) un periodo para sentirse en el medio, entre la adolescencia y la adultez.

El reporte de los participantes sustenta la importancia de la exploración general que se realiza en la Universidad, la cual les permite avanzar paulatinamente en la construcción y luego consolidación de la identidad personal, proceso propio de la adolescencia que se mantiene vigente en los primeros años de esta etapa de vida (Arnett, 2000, 2004). Así lo plantean Tanner y Arnett (2009), a través de su concepto de Re-centramiento, el cual hace referencia a la tarea psicosocial propia de la Adultez Emergente, un proceso de tres etapas que alude a la inter-dependencia del desarrollo, y la consideración del individuo en un contexto. Así, los adultos emergentes en una primera y segunda etapa transitarían desde la adolescencia a la adultez emergente siendo sujetos de cambios en la dinámica de la dependencia/ independencia en sus relaciones y su identidad, explorando en posibles roles y relaciones interpersonales, no percibiéndose preparados para compromisos estables y duraderos. Posiblemente esto último se relacione con la complejidad con que los jóvenes perciben la adultez, observando el mundo adulto con cierto temor y suspicacia por las altas responsabilidades que le atribuyen y para las cuales no se sienten preparados, generándose cierta confusión en la autodefinición además de fomentar la permanencia en procesos adolescentes tales como la confusión de roles, cierta dependencia familiar y baja autonomía (Kroger, 2007). Esto ocurre en los primeros dos años universitarios, pues con el paso del tiempo los estudiantes reportan sentirse más maduros y adquirir progresivamente mayor autonomía, lo cual favorece su disposición a enfrentar la adultez, dando paso a la tercera etapa de Re-centramiento, en donde los jóvenes van asumiendo compromisos, roles y responsabilidades duraderas propias del adulto (Tanner y Arnett, 2009).

Otro hallazgo importante es que los participantes explicitaron espontáneamente contenidos relacionados con Identidad y Autoconcepto, Relación con los padres y salida del hogar, y Amor y sexualidad. Estos aspectos, al no estar contenidos en la teoría original, podrían constituir un núcleo de contenidos específicos de la cultura chilena, la cual, a pesar de los cambios demográficos descritos previamente, mantiene aspectos vinculados al colectivismo, sobre todo en el ámbito de las relaciones familiares e interpersonales como se analizará a continuación (Novoa y Barra, 2015; Rojas-Méndez et al., 2008).

A este respecto se observa que los jóvenes transitan en dos procesos que no son excluyentes, por una parte están centrados en sí mismos, en su identidad, sus experiencias individuales y en el desarrollo de la autonomía, y por otra desarrollan y mantienen los vínculos relacionales que han construido o que están construyendo en esta etapa, específicamente con la familia, los amigos, y en algunos casos una relación de pareja. Esta interdependencia se ha estudiado en las culturas minoritarias de Estados Unidos como los latinos, los afroamericanos o los orientales, grupos en los cuales pueden haber razones económicas para permanecer en el hogar, y al mismo tiempo razones culturales como el gran énfasis en la cercanía de la familia, y menos énfasis en ser independiente como un valor por sí mismo (Fuligni y Tseng, 1999; Xia, Ko, y Xie, 2013). La importancia de los vínculos familiares también ha sido reportada en estudios con población latina (Stein, González, Cupito, Kiang, y Supple, 2013), a través del Familismo, concepto que hace referencia a un valor cultural presente en la cultura latina, que dicta las normas, expectativas y creencias acerca de la familia, y se compone de lealtad y respeto por el grupo familiar. Así, los jóvenes universitarios chilenos transitan por el desafío de construir y consolidar su identidad, y al mismo tiempo necesitan mantener el contacto y el cuidado del vínculo directo con su familia. Si salen del hogar familiar a una edad temprana mantienen los lazos con su grupo de origen, su adaptación universitaria depende en parte de la cercanía con su familia, y como reportaron, se sienten presionados internamente a obtener logros académicos adecuados como tributo al apoyo familiar económico y afectivo en la etapa universitaria.

En relación con la conformación de pareja, los universitarios chilenos se asemejan a sus pares estadounidenses. En general, no tienen como objetivo inmediato formar una relación de pareja estable y pueden involucrarse en relaciones inestables con distinto grado de compromiso (Shulman y Connolly, 2013). Una relación de pareja significa construir una intimidad con un otro, esto es, requerimientos básicos de tiempo y privacidad, compartir tiempo, auto-revelación y confianza (Palma, 2006) aspectos claves para los cuales probablemente aún no se sienten preparados.

En el área de la sexualidad, las investigaciones refieren que en los jóvenes chilenos están presentes los encuentros 
esporádicos o casuales con diversos grados de intimidad sexual, sin compromiso futuro, que pueden establecerse con una o varias personas (Rivera, Cruz, y Muñoz, 2011). Sin embargo, es llamativo que este tema no pudiera explorarse abiertamente con los participantes; es probable que el formato de grupo focal hubiera aumentado esta dificultad, pues la presentación cara a cara para tratar temas íntimos aumenta la deseabilidad de las respuestas y el temor al juicio externo sobre las respuestas brindadas (INJUV, 2012).

El mayor aporte de este trabajo fue haber explorado las características de la Adultez Emergente chilena en universitarios, recogiendo aspectos propios de la cultura que no habían sido considerados en la teoría original, entregando una visión comprehensiva y descriptiva de este grupo de jóvenes en el momento en que esta generación reclama un rol más protagónico en la sociedad. Además, da cuenta de los desafíos y oportunidades en la etapa universitaria, la cual al ser una etapa clave afecta las posibilidades de desarrollo de los jóvenes, y a su vez, las posibilidades de desarrollo de la sociedad (INJUV, 2012).

Este estudio presenta limitaciones, pues reporta solo los aspectos cualitativos recogidos en una primera etapa de investigación la cual debe complementarse con la sistematización cuantitativa de estos hallazgos, que permita dar cuenta los aspectos generales y culturales del constructo de AE en Chile. Otra limitación es el haber trabajado con una muestra solo de universitarios, lo cual restringe la generalización de los hallazgos. Futuras líneas de investigación podrían incluir otros grupos como jóvenes que no se han enrolado en la universidad (por ejemplo, jóvenes trabajadores), a fin de estudiar si las características generales y culturales de la AE están presentes en distintos grupos de jóvenes en nuestro contexto sociocultural.

Finalmente, los adultos emergentes universitarios chilenos se encuentran en una etapa de transición, centrados en sí mismos, pero también en sus relaciones familiares y sociales, interesados en explorar la diversidad que se les presenta, y para ellos, aún no es tiempo para avanzar a la adultez, pues transitan por la etapa universitaria lentamente, sin apuros, viviendo el presente y sus procesos psicológicos asociados.

\section{Referencias}

Arnett, J. J. (2000). Emerging adulthood: A theory of development from the late teens through the twenties. American Psychologist, 55, 469-480.

Arnett, J. J. (2004). Adolescence and emerging adulthood: A cultural approach (2nd ed.). Upper Saddle River, New Jersey: Pearson.

Arnett, J. J. (2012). The Clark University Poll of Emerging Adults. Recuperado desde http://www.clarku.edu/clark-poll-emerging-adults/
Arnett, J. J., Kloep, M., Hendry, L., y Tanner, J. (2011). Debating Emerging Adulthood, Stage or Process? New York: Oxford University Press.

Baader, T., Rojas, C., Molina, J., Gotelli, M., Alamo, C., Fierro, C., Venezian, S., y Dittus, P. (2014). Diagnóstico de la prevalencia de trastornos de la salud mental en estudiantes universitarios y los factores de riesgo emocionales asociados. Revista chilena de neuro-psiquiatría, 52, 167-176.

Casas, F., Alfaro, J., Figuer, C., Valdenegro, B., Crous, G., y Oyarzún, D. (2014). Bienestar subjetivo y trabajo en jóvenes universitarios: estudio comparativo entre Chile y España. Revista Psicologia Organizações e Trabalho, 14, 381-393.

Cerda, R. (2008). Cambios demográficos y sus impactos en Chile. Estudios Públicos, 110, 1-75.

Consejo Nacional de Educación, CNED (2015). Matricula total de Educación Superior, años 2005-2015. Recuperado desde http://www. cned.cl/public/Secciones/SeccionIndicesPostulantes/CNED_IndicesTablInd MatriculaSistema.html?IdRegistro $=001$

Dávila, O., y Ghiardo, F. (2012). Transiciones a la vida adulta: Generaciones y Cambio Social en Chile. Última Década, 37, 69-83.

Fuligni, A. J., y Tseng, V. (1999). Family obligations and the academic motivation of adolescents from inmigrant and American-born families. Advances in Motivation and Achievement, 11, 159-183.

Glaser, B., y Strauss, A. (1967). The Discovery of Grounded Theory: strategies for qualitative research. New York: Aldine.

Instituto Nacional de Estadísticas, INE (2011). Estadísticas vitales, Informe Anual 2011. Recuperado desde http://www.ine.cl/canales/menu/publicaciones/calendario_de_publicaciones/pdf/cocomple_vitales_2011.pdf

Instituto Nacional de la Juventud, INJUV (2012). Séptima Encuesta Nacional de la Juventud 2012. Recuperado desde http://www.injuv.gob. $\mathrm{cl} /$ portal/wp-content/files_mf/septimaencuestanacionaljuventud2.pdf

Instituto Nacional de la Juventud, INJUV (2016). Boletín Electrónico interno Area de Estudios $N^{\circ} 2$. Recuperado desde http://www.injuv.gob. $\mathrm{cl} /$ portal/wp-content/files $\mathrm{mf} /$ boletinestudios20162.pdf

Kroger, J. (2007). Identity development: Adolescence through adulthood. Thousand Oaks, CA: SAGE Publications

Mediano-Stoltze, F., Repetto, P., y Molina, Y. (2013). Insatisfacción corporal, creencias sobre control del peso y consumo de cigarrillos en jóvenes. Terapia Psicológica, 31, 155-163.

Micin, S., y Bagladi, V. (2011). Salud Mental en Estudiantes Universitarios: Incidencia de Psicopatología y antecedentes de conducta suicida en población que acude a un Servicio de Salud Estudiantil. Terapia Psicológica, 29, 53-64.

Morales, G., del Valle, C., Belmar, C., Orellana, Y., Soto, A., e Ivanovic, D. (2011). Prevalencia de consumo de drogas en estudiantes universitarios que cursan primer y cuarto año. Revista Médica de Chile, 139, $1573-1580$

Novoa, C., y Barra, E. (2015). Influencia del apoyo social percibido y los factores de personalidad en la satisfacción vital de estudiantes universitarios. Terapia Psicológica, 33, 239-245.

Palma, I. (2006) Trayectorias sexuales, vínculos y contextos en la generación que hace su entrada en la sexualidad activa en democracia. Revista Observatorio de Juventud, Instituto Nacional de la Juventud, INJUV, 3, 9-26.

Pérez, J. C., Cumsille, P., y Martínez, M. (Marzo, 2008). Construct Validity of the Inventory of Dimensions of Emerging Adulthood in a Chilean Sample. Póster presentado en Society for Research on Adolescence Biennial Meeting, Chicago, Illinions, USA.

Reifman, A., Arnett, J., y Colwell, M. (2007). Emerging adulthood: Theory, assessment, and application. Journal of Youth Development, 2, 39-50.

Rivera, D., Cruz, C., y Muñoz, C. (2011). Satisfacción en las Relaciones de Pareja en la Adultez Emergente: El Rol del Apego, la Intimidad y la Depresión. Terapia Psicológica, 29, 77-83.

Rojas-Méndez, J., Coutiño-Hill, V., Bhagat, R., y Moustafa, K. (2008). Evaluación del Individualismo y Colectivismo Horizontal y Vertical en la Sociedad Chilena. Multidisciplinary Business Review, 1, 36-48.

Shulman, S., y Connolly, J. (2013). The Challenge of Romantic Relationships in Emerging Adulthood : Reconceptualization of the Field. Emerging Adulthood, 1, 27-39. 
Stein, G. L., Gonzalez, L. M., Cupito, A. M., Kiang, L., y Supple, A. J. (2013). The protective role of Familism in the lives of Latino Adolescents. Journal of Family Issues. doi: 10.1177/0192513X13502480

Tanner, J. L., y Arnett J. J. (2009). The emergence of "emerging adulthood": The new life stage between adolescence and young adulthood. In A. Furlong (Ed.), Handbook of youth and young adulthood (pp. 39-48). New York: Routledge.

Véliz-Burgos, A., y Apodaca, P. (2012). Niveles de Autoconcepto, Autoeficacia Académica y Bienestar Psicológico en Estudiantes Universitarios de la ciudad de Temuco. Salud y Sociedad, 3, 131-150.

Xia, Y. R., Ko, K. A., y Xie, X. (2013). The adjustment of Asian American families to the U.S. context: The ecology of strengths and stress. En G. W. Peterson y K. R. Bush (Eds.), Handbook of marriage and the family (pp. 705-722). New York, NY: Springer. 